BULLETIN OF THE

AMERICAN MATHEMATICAL SOCIETY

Volume 79, Number 3, May 1973

\title{
STABLE RINGS AND A PROBLEM OF BASS
}

\author{
BY JUDITH D. SALLY AND WOLMER V. VASCONCELOS \\ Communicated by Hyman Bass, November 20, 1972
}

In [1] Bass proved the following theorem. Let $A$ be a 1-dimensional Noetherian ring with finite integral closure. ${ }^{1}$ If every ideal of $A$ can be generated by two elements, then every ideal is projective over its endomorphism ring. Bass also conjectured that the converse holds. Theorem 2.4 below shows that this is indeed the case.

1. Stable ideals and stable rings. Let $A$ be a commutative Noetherian ring with 1 . In analogy with Lipman's terminology in [3], we call an ideal $I$ of a ring $A$ stable if $I$ is projective over its endomorphism ring, $\operatorname{End}_{A}(I)$. The ring $A$ is stable if every ideal of $A$ is stable. Since the relationship between stability and multiplicity is one key factor in the proof of the theorem, we gather some of the needed facts here.

Let $I$ be an ideal of $A$ which contains a nonzero divisor. Then $\operatorname{End}_{A}(I)$ $\cong(I: I)=\{x \in K \mid x I \subseteq I\}$, where $K$ is the total quotient ring of $A$. Thus End $_{A}(I)$ is a subring of the integral closure of $A$ in $K$ and $I$ is an ideal of $\operatorname{End}_{A}(I)$.

Suppose that $A$ is a 1-dimensional local Macaulay ring and that $I$ is an ideal containing a nonzero divisor. Then $I$ is a stable ideal of $A$ if and only if $I$ is a principal ideal of the semilocal ring $\operatorname{End}_{A}(I)$. Therefore, if $I$ is stable, $\operatorname{End}_{A}(I)=A^{I}$, the ring obtained from $A$ by blowing up $I$ (cf. [3]). For reference, we state as Proposition 1.1 the characterization of stable ideals due to Lipman [3]. The notation is as follows: $\mu(I)$ denotes the multiplicity of an ideal $I$ and $\lambda(B)$ denotes the length of an $A$-module $B$.

Proposition 1.1 (LipMAN). Let $A$ be a 1-dimensional local Macaulay ring. The following conditions are equivalent for an ideal I containing a nonzero divisor.

(1) I is stable;

(2) $\lambda\left(I^{n} / I^{n+1}\right)=\mu(I)$, for all $n>0$;

(3) $\lambda\left(A / I^{n}\right)=\mu(I) n-\lambda((I: I) / A)$, for all $n>0$.

2. Proof of Bass' conjecture. We will need the following proposition which turns out to be a special case of a theorem of Rees [5]. We give a simple direct proof.

Proposition 2.1. Let $A$ be a 1-dimensional local Macaulay ring with

AMS (MOS) subject classifications (1970). Primary 13H10, 13H15.

${ }^{1}$ Here, and in the statement of Theorem $2.4, A$ is assumed to have no nonzero nilpotent elements.

Copyright (C) American Mathematical Society 1973 
maximal ideal $\mathrm{m}$. If $I$ is any ideal of $A$ which contains a nonzero divisor, then

$$
v(I) \leqq \mu(A),
$$

where $v(I)$ denotes the number of elements in a minimal basis of $I$ and $\mu(A)$ is the multiplicity of $A$.

Proof. By passing to $A(X), \mathfrak{m} A(X)$ and $I A(X)$ as in $[4, \mathrm{pp} .18,71]$, we may assume that $A / \mathrm{m}$ is an infinite field. Therefore, $\mathrm{m}$ has a transversal element $x$, i.e., there is a nonzero divisor $x$ in $m$ such that $x \mathrm{~m}^{n}=\mathrm{m}^{n+1}$, for some $n>0$. Let $I$ be any ideal containing a nonzero divisor. Then $\lambda(A / x A)=\lambda(I / x I)=\mu(A)$. For, $\lambda(A / x A)=\lambda(A / x A)+\lambda(x A / x I)-\lambda(A / I)$ $=\lambda(A / x I)-\lambda(A / I)=\lambda(I / x I)$ gives the first equality, and if we take $I=\mathfrak{m}^{k}$ for any $k \geqq n$, we have $\lambda(A / x A)=\lambda\left(\mathfrak{m}^{k} / \mathfrak{m}^{k+1}\right)=\mu(A)$. The exact sequence

$$
0 \rightarrow \mathfrak{m} I / x I \rightarrow I / x I \rightarrow I / \mathfrak{m} I \rightarrow 0
$$

gives $\lambda(I / \mathfrak{m} I)=\lambda(I / x I)-\lambda(\mathfrak{m} I / x I)=\mu(A)-\lambda(\mathfrak{m} I / x I)$. Hence, $\lambda(I / \mathfrak{m} I)$ $=v(I) \leqq \mu(A)$.

COROLlary 2.2. If, in addition, $m$ is a stable ideal of $A$, then

$$
v(I) \leqq v(m)
$$

for all ideals $I$ which contain a nonzero divisor.

Proof. By Proposition 1.1, $v(\mathfrak{m})=\lambda\left(\mathfrak{m} / \mathfrak{m}^{2}\right)=\mu(\mathfrak{m})=\mu(A)$.

Proposition 2.3. Let $A$ be a 1-dimensional Gorenstein ring in which maximal ideals are not minimal primes. If all maximal ideals of $A$ are stable, then every ideal of $A$ which contains a nonzero divisor can be generated by two elements.

Proof. By [1, Lemma 7.4] or [6] we may assume that $A$ is local with maximal ideal $\mathrm{m}$. We apply Proposition 1.1 to the stable ideal $\mathrm{m}$ and obtain $\lambda(A / \mathfrak{m})=\mu(A)-\lambda((\mathfrak{m}: \mathfrak{m}) / A)=\mu(A)-\lambda\left(\mathfrak{m}^{-1} / A\right)$. Since $A$ is Gorenstein, $\operatorname{dim}_{A / \mathfrak{m}}\left(\operatorname{Ext}_{A}^{1}(A / \mathfrak{m}, A)\right)=\lambda\left(\mathfrak{m}^{-1} / A\right)=1$. Thus, $\mu(A)=v(\mathfrak{m})=$ 2 and the desired result follows from Proposition 2.1.

Remark 1. Under the conditions of Proposition 2.3, it can be shown that all ideals can be generated by two elements and that all ideals are stable.

REMARK 2. If $A$ is a reduced ring, then an upper bound for the number of generators of ideals which contain a nonzero divisor is an upper bound for the number of generators of all ideals. This follows from consideration of the ideal $I+$ ann $I=I \oplus$ ann $I$.

To prove Bass' conjecture it is now sufficient just to prove that the ring in question is Gorenstein. This is accomplished by means of the existence and properties of the canonical ideal as in [2]. 
THEOREM 2.4. Let $A$ be a 1-dimensional ring with finite integral closure. If $A$ is stable, then every ideal of $A$ can be generated by two elements.

Proof. Since $A$ is reduced, we need only consider ideals $I$ which contain a nonzero divisor. Furthermore, we may assume that $A$ is local with maximal ideal m. $\hat{A}$, the completion of $A$, is reduced. We apply [2, Satz 6.21 ] to assert that $A$ has a canonical ideal $\mathfrak{f}$. Now a canonical ideal $\mathfrak{f}$ has the following properties:

(i) $(\mathfrak{f}: \mathfrak{f})=A$,

(ii) $v(\mathfrak{f})=\operatorname{dim}_{A / \mathfrak{m}}\left(\operatorname{Ext}_{A}^{1}(A / \mathfrak{m}, A)\right)=\lambda\left(\mathfrak{m}^{-1} / A\right)$.

Since $A$ is stable, $\mathfrak{f}$ is a stable ideal. Thus, (i) implies that $\mathfrak{f}$ is a principal ideal of $A$, i.e., $v(\mathfrak{t})=1$, and (ii) implies that $A$ is a Gorenstein ring. The theorem now follows from Proposition 2.3.

REMARK 3. In the result above it is enough to assume that each localization of $A$ at a maximal ideal has finite integral closure, or, more generally, that locally $A$ admits a canonical ideal.

\section{REFERENCES}

1. H. Bass, On the ubiquity of Gorenstein rings, Math. Z. 82 (1963), 8-28. MR 27 \# 3669.

2. J. Herzog and E. Kunz (Editors), Der kanonische Modul eines Cohen-Macaulay-Rings, Lecture Notes in Math., vol. 238, Springer-Verlag, Berlin and New York, 1971.

3. J. Lipman, Stable ideals and Arf rings, Amer. J. Math. 93 (1971), 649-685. MR 44 \#203.

4. M. Nagata, Local rings, Interscience Tracts in Pure and Appl. Math., no. 13, Interscience, New York, 1962. MR 27 \# 5790.

5. D. Rees, Estimates for the minimum number of generators of modules over CohenMacaulay local rings (preprint).

6. R. Swan, The number of generators of a module, Math. Z. 102 (1967), 318-322. MR 36 \# 1434.

Department of Mathematics, Rutgers University, New Brunswick, New Jersey 08903 (Current address of Wolmer V. Vasconcelos)

Department of Mathematics, NORThWESTERN University, Evanston, Illinois 60201 (Current address of Judith Sally) 